\title{
PREPARAÇÃO DE NOVOS FILMES POLIMÉRICOS CONTENDO NANOEMULSÕES DO ÓLEO DE MELALEUCA, COPAÍBA E LIMÃO PARA APLICAÇÃO COMO BIOMATERIAL
}

\author{
Viviane G. A. Pires e Márcia R. de Moura* \\ Departamento de Física e Química, Faculdade de Engenharia de Ilha Solteira, Universidade Estadual Paulista "Júlio de Mesquita \\ Filho", 15385-000 Ilha Solteira - SP, Brasil
}

Recebido em 28/01/2016; aceito em 07/07/2016; publicado na web em 20/07/2016

\begin{abstract}
PREPARATION OF NEW NANOCOMPOSITES CONTAINING NANOEMULSIONS OF MELALEUCA, COPAÍBA AND LEMON OIL FOR APPLICATION AS BIOMATERIAL. The sodium alginate is a linear polymer used in industry and in medicine for many applications such as dressings for wounds, due to low toxicity and maintains a proper moist environment. The essential oils are materials that have antimicrobial activity and are active materials, which may be incorporated in polymeric films. This study has as objective the produce alginate films incorporated with nanoemulsions of melaleuca, copaiba and lemon oil and investigate water vapor permeability (WVP) analysis of the films, particle size and $\zeta$-potential of nanoemulsions. Particle size analyzes showed formation of large and small particles nanoemulsion depending on the agitation speed used during production of nanoemulsions. Results of the WVP analyzes showed that the film with alginate $3 \% \mathrm{w} / \mathrm{v}$ and nanoemulsions with little particle prepared with agitation speed of $15000 \mathrm{rpm}$ showed better WVP. Produced films show good quality for a future application as dressings because it has good WVP. This is very interesting because the skin needs to breathe.
\end{abstract}

Keywords: nanoemulsions; sodium alginate; melaleuca oil; copaiba oil; lemon oil.

\section{INTRODUÇÃO}

O alginato de sódio ( $\mathrm{NaAlg}$ ) é um polissacarídeo proveniente da metabolização do ácido algínico, encontrado na parede celular de algas marinhas marrons, em diversas formas de sais catiônicos da água do mar, como sais de cálcio, sódio e potássio. ${ }^{1}$ Além disso, é um copolímero aniônico linear formado por unidades monoméricas de 1,4-ß-D-manurônicos (M) e 1,4- $\alpha$-L-gulurônicos (G), o qual tem sido muito utilizado em pesquisas científicas e têm mostrado seu potencial antioxidante e de biocompatibilidade. ${ }^{1,2}$

Não apresenta atividade antimicrobiana, entretanto, sua utilização na forma de curativos para feridas, suportes e revestimentos em diversas áreas, como médicas, farmacêuticas, alimentícias entre outras, tem se mostrado eficaz devido à presença de uma barreira seletiva que mantém o controle de umidade, trocas gasosas com o ambiente, capta o oxigênio e preserva o sabor e o aroma dos produtos. ${ }^{3}$ Em 1948 , Passe $e t a l .{ }^{4}$ já falavam em suas pesquisas sobre as vantagens da utilização de alginato como curativo, e atualmente tem crescido o número de pesquisas nesta área, ${ }^{5}$ tornando-se necessário o desenvolvimento de novos materiais os quais apresentem as propriedades do alginato somadas com as atividades antimicrobianas necessárias para sua utilização no tratamento de feridas.

Os materiais que apresentam atividade antimicrobiana são materiais ativos, como é o caso dos óleos essenciais, que podem ser incorporados em filmes poliméricos ajudando no processo de melhora da pele lesionada, evitando a infecção e inflamação da ferida, e apresentando em sua composição substâncias antifúngicas e antibacterianas que auxiliem na cicatrização. ${ }^{6}$

Os óleos essenciais (OEs) são substâncias oleosas aromáticas obtidas através de material vegetal, que apresentam terpenos e fenólicos em sua composição, os quais, nos últimos anos, vêm sendo estudados devido ao seu uso medicinal e comercial em diversos produtos higiênicos e drogarias devido à erradicação das bactérias. ${ }^{7,8}$

Um dos óleos que tem mostrado em diversas pesquisas suas

*e-mail: marcia@dfq.feis.unesp.br atividades antibacteriana e anti-inflamatória é o óleo de melaleuca, também conhecido como árvore do chá. Tendo sua planta nativa na Austrália, o óleo de melaleuca também tem sido encontrado ao redor do mundo, inclusive no Brasil. ${ }^{9-11} \mathrm{O}$ óleo de melaleuca é um líquido límpido que apresenta odor distinto, rico em monoterpenos, sendo que, dentre seus mais de 100 componentes, o mais abundante é o terpinen-4-ol, que compõe aproximadamente $40 \%$ do óleo. Estudos feitos com o óleo da árvore do chá mostraram efetividade contra diversas doenças, dentre elas acnes superficiais, candidíase oral, entre outras. ${ }^{12}$

Outro óleo que tem mostrado sua eficácia no tratamento de feridas e no processo de cicatrização desde a época em que o Brasil era colonizado pelos portugueses é o óleo de copaíba. Este líquido transparente que varia da coloração amarela a marrom foi descoberto primeiro pelos índios no tratamento de machucados, e difundido pela Europa através dos colonizadores. Hoje, através de estudos e pesquisas, são comprovadas suas atividades farmacológicas, apresentando propriedades anti-inflamatórias e cicatrizantes. ${ }^{13-15}$

Além dos óleos citados acima, o óleo de limão do gênero Citrus, formado por hidrocarbonetos, compostos oxigenados, cera, parafina e limoneno (50 a $80 \%$ do total), assim como outros cítricos, é utilizado em bebidas, produtos de higiene e cosméticos. No entanto, algumas de suas propriedades têm chamado a atenção de pesquisas médicas, devido às atividades antimicrobianas, antifúngica, antibacteriana, antiviral e antiparasitária, que faz com que este óleo seja considerado de grande eficácia na utilização em filmes curativos com propriedade ativa. ${ }^{16,17}$

Devido às propriedades hidrofóbicas dos óleos, há a necessidade de formar nanoemulsões deles em água, melhorando assim sua homogeneidade e dispersão nos filmes. As vantagens das nanoemulsões estão no tamanho das partículas (escala nanométrica), as quais melhoram suas propriedades físicas, químicas, além de aumentar as atividades biológicas do óleo nos filmes, devido a melhor distribuição do mesmo na superfície. ${ }^{18,19}$

Sendo assim, o presente trabalho tem como finalidade desenvolver filmes poliméricos incorporados com óleos essenciais através 
de nanoemulsões de óleo em água, ou seja, formar um sistema de dispersão do óleo na água utilizando dispositivos mecânicos como fonte de energia, e estudar se a incorporação de nanoemulsões dos óleos em uma matriz polimérica influencia na Permeabilidade de Vapor de Água, uma vez que este trabalho tem como intuito uma futura aplicação dos filmes como curativos.

\section{PARTE EXPERIMENTAL}

\section{Preparação das nanoemulsões}

As nanoemulsões foram produzidas pela agitação das soluções de $1 \%$ de óleos essenciais de melaleuca, limão, copaíba (Ferquima) e $0,75 \%$ de Tween 80 (Sigma) em água. As soluções foram agitadas em um homogeneizador Ultra-Turrax (Marconi-MA102). As velocidades de agitação foram de 12000 e $15000 \mathrm{rpm}$ no tempo de 5 minutos. As suspensões obtidas foram avaliadas visualmente: presença de sedimentados ou não, opalescência e homogeneidade da solução e separação ou não de fase após período de repouso.

\section{Tamanho médio das partículas nas nanoemulsões}

Através do aparelho Zetasizer Nano Series (Malvern Instruments Inc. USA) foram realizadas as medidas de tamanho médio das partículas presentes nas nanoemulsões. As análises foram feitas utilizando um índice de refração de 1,590 e comprimento de onda do laser de $632,8 \mathrm{~nm}$. Todas em triplicata na temperatura de $25^{\circ} \mathrm{C}$.

\section{Preparação dos filmes}

Os filmes foram produzidos através da técnica de casting. $\mathrm{O}$ alginato de sódio foi dissolvido nas nanoemulsões sob agitação. A solução viscosa resultante foi agitada até completa dissolução do alginato e deixada em repouso por 24 horas para eliminação de bolhas. A variação do tipo de filmes se deu pela variação da concentração de alginato $(2 \%$ e $3 \% \mathrm{~m} / \mathrm{v})$, pela diferença de síntese das nanoemulsões (12.000 rpm e $15.000 \mathrm{rpm}$ ) e pela variação de temperatura na secagem $\left(30\right.$ e $\left.45^{\circ} \mathrm{C}\right)$.

\section{Determinação da espessura}

As espessuras dos filmes foram determinadas utilizando um micrômetro digital (No. 7326, Mitutoyo Manufacturing, Japan). Foram realizadas 3 análises em diferentes posições do filme seco. $\mathrm{O}$ micrômetro utilizado apresentava precisão e $0,001 \mathrm{~mm}$.

\section{Microscopia Eletrônica de Varredura (MEV)}

As análises da morfologia dos filmes foram feitas usando um microscópio eletrônico de varredura computadorizado da marca ZEISS modelo EVO LS15, com voltagem de $12 \mathrm{kV}$. Os filmes foram fixados em uma fita de carbono dupla face e colocadas em um metalizador (Quorum - Q150 T) durante um minuto e meio para que houvesse a deposição de uma fina camada de ouro sobre eles.

\section{Permeabilidade ao vapor de água (WVP)}

As análises foram realizadas utilizando o método adaptado da norma ASTM E96-80 ${ }^{20}$ descrito na literatura, ${ }^{21}$ como realizado em outros trabalhos. ${ }^{22}$ Os filmes foram cortados na forma de um círculo com $5 \mathrm{~cm}$ de diâmetro e colocados em cima de placas de Teflon ${ }^{\circledR}$, as quais comportavam $6 \mathrm{~mL}$ de água destilada. Foi colocada uma segunda placa por cima da primeira e ambas foram fixadas por parafusos.
Foram realizadas três análises de WVP para cada filme. As placas com os filmes foram colocadas em um ambiente de temperatura controlada $\left(25^{\circ} \mathrm{C}\right)$ (estufa) e foi utilizada sílica para o controle da umidade interna. Foram feitas pesagens periódicas das placas e os valores das massas anotados para utilização na determinação da WVP.

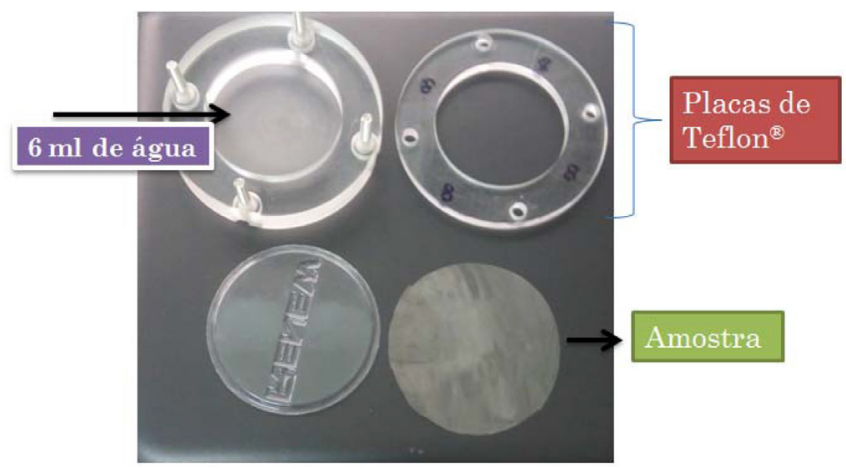

Figura 1. Amostra de filme e as placas de Teflon utilizadas nas análises de WVP

Através dos valores de WVTR (Velocidade de transmissão de vapor de água), foi determinado o valor de WVP para os filmes, utilizando-se as Equações de 1 a 3 abaixo, em que: mw é a massa molar da água $\left(18 \mathrm{~g} \mathrm{~mol}^{-1}\right)$, D é a difusividade do vapor de água pelo ar a $298 \mathrm{~K}\left(0,102 \mathrm{~m}^{2} \mathrm{~s}^{-1}\right)$, P é a pressão total (1 atm), $\mathrm{p}_{1}$ é a pressão de vapor a $298 \mathrm{~K}$, R é a constante do gases $\left(82,1 \times 10^{-6} \mathrm{~m}^{3} \mathrm{~atm} \mathrm{~g}^{-1} \mathrm{~mol}^{-1}\right.$ $\mathrm{K}^{-1}$ ), z é a altura média que o gás inerte atinge, $p_{2}$ (pressão parcial de vapor da parte inferior do filme), $p_{3}$ (pressão parcial de vapor da parte superior do filme) e $y$ (espessura média dos filmes).

$$
\begin{aligned}
& \begin{array}{l}
\text { Velocidade de transmissão de } \\
\text { vapor de água }(\text { WVTR })
\end{array}=\frac{\text { perda de massa do filme }}{\text { área do filme }} \\
& \qquad \begin{array}{l}
\text { WVTR }=\frac{\text { mW.P.D. } \ln \left[\left(\mathrm{p}-\mathrm{p}_{2}\right) /\left(\mathrm{p}-\mathrm{p}_{1}\right)\right]}{\text { R.T.z }} \\
\mathrm{WVP}=\frac{\mathrm{WVTR}}{\left(\mathrm{p}_{2}-\mathrm{p}_{3}\right)} y
\end{array}
\end{aligned}
$$

\section{RESULTADOS E DISCUSSÕES}

A Figura 2 representa uma partícula de nanoemulsão na qual as moléculas do tensoativo (Tween 80) estão organizadas na interface óleo/água através das interações ocorrentes entre suas regiões: hidrofóbica (apolar) com o óleo (apolar) e hidrofílicas (polar) com a água (polar)

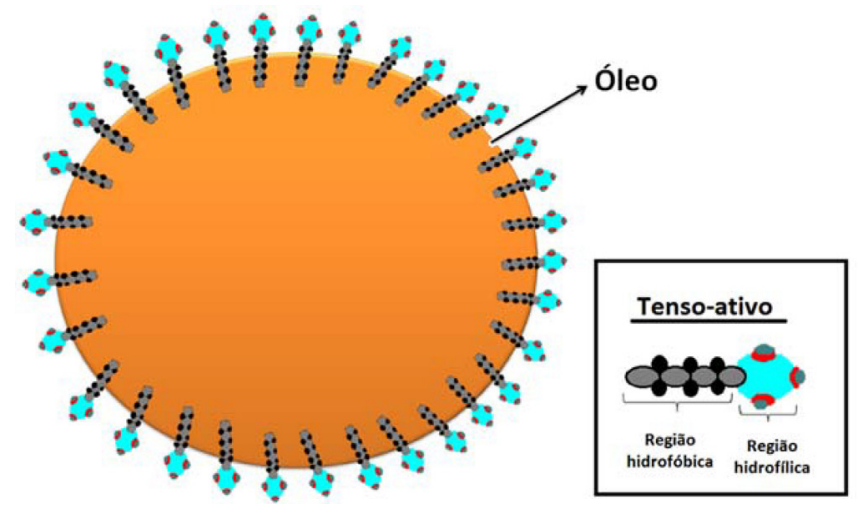

Figura 2. Figura representativa da partícula de nanoemulsão 
A Figura 3 mostra o tamanho médio das gotículas de óleo em função da velocidade de rotação usada no preparo das nanoemulsões. Nota-se claramente que quanto maior a velocidade de rotação, menor é o tamanho médio das gotículas de óleo.

O potencial zeta é um reflexo do potencial de superfície das gotículas de nanoemulsões, podendo ser influenciado por mudanças da interface com o meio dispersante. ${ }^{23}$ Neste trabalho, os potenciais zeta das nanoemulsões dos óleos são mostrados na Tabela 1 , nela é possível observar resultados positivos, ou seja, os potenciais zeta das partículas de nanoemulsões apresentam valores em módulo maiores que $20 \mathrm{mV}$ e próximos de $25 \mathrm{mV}$, com exceção das nanoemulsões com óleo de melaleuca preparadas a $15000 \mathrm{rpm}$.

Esses resultados são importantes, pois o alto valor de potencial zeta é um indicativo de que as gotículas das nanoemulsões têm forças de repulsão fortes o suficiente para impedir sua coalescência, mantendo sua estabilidade. ${ }^{24}$

Como o tensoativo (Tween 80) utilizado para estabilizar as gotículas de óleo foi um não iônico, sugere-se que os valores negativos de potencial zeta são resultados da adsorção de íons hidroxilas na interface óleo/água provenientes das ligações de hidrogênio da água com os grupamentos químicos presentes no Tween 80 e nos compostos dos óleos essenciais, tornando a carga superficial da gotícula de nanoemulsão mais negativa. ${ }^{25}$

Tabela 1. Potencial zeta das nanoemulsões dos óleos essenciais estudados

\begin{tabular}{ccc}
\hline Nanoemulsões & $\begin{array}{c}\text { Velocidade de } \\
\text { agitação }\end{array}$ & Potencial zeta \\
\hline Óleo de Limão & $12000 \mathrm{rpm}$ & $-24,6 \pm 0,6$ \\
Óleo de Limão & $15000 \mathrm{rpm}$ & $-21,0 \pm 0,9$ \\
Óleo de Copaíba & $12000 \mathrm{rpm}$ & $-24,7 \pm 1$ \\
Óleo de Copaíba & $15000 \mathrm{rpm}$ & $-26,8 \pm 2$ \\
Óleo de Melaleuca & $12000 \mathrm{rpm}$ & $-26,8 \pm 1$ \\
Óleo de Melaleuca & $15000 \mathrm{rpm}$ & $-18,2 \pm 0,7$ \\
\hline
\end{tabular}

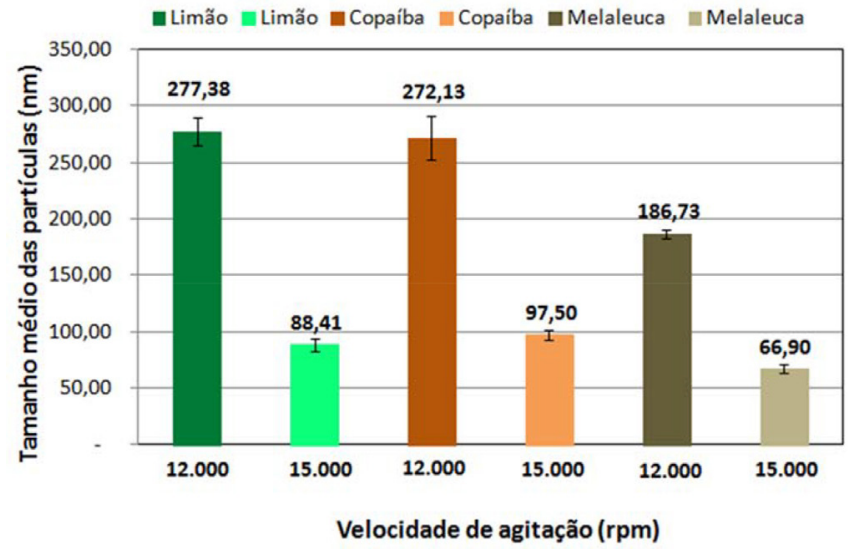

Figura 3. Valores de tamanho médio das nanoemulsões em função da velocidade de agitação

A produção de partículas menores de nanoemulsões ocorreu devido à alta velocidade de rotação, que fez com que as moléculas de óleo se dispersaram mais, formando nanoemulsões de aglomerados lipofílicos menores. Já as menores rotações não dispersaram tão bem as moléculas de óleo, fazendo com que elas tendessem à aglomeração, resultando em nanoemulsões com uma parte lipofílica maior.

Com o intuito de descobrir as melhores condições para a produção dos filmes, foi realizado um planejamento fatorial $2^{3} \mathrm{e}$, em seguida, uma avaliação subjetiva, para classifica-los segundo sua continuidade (fratura ou ruptura após secagem), homogeneidade (partículas visíveis a olho nu, opacidade ou diferença de cores) e manuseabilidade (facilidade do filme em se romper). ${ }^{21}$

O planejamento fatorial $2^{3}$ foi realizado apresentando 2 níveis $(+\mathrm{e}$ -) e 3 variáveis (A, B e C): em que a variável A representava a porcentagem de alginato $(\mathrm{m} / \mathrm{v})$, a B o tamanho da partícula de nanoemulsão (maiores ou menores, de acordo com a velocidade de rotação) e $\mathrm{C}$ a temperatura de secagem. O nível superior $(+)$ representava maiores valores trabalhados e nível inferior (-) menores valores trabalhados, como mostrado na Tabela 2.

Tabela 2. Planejamento fatorial $2^{3}$ para aquisição dos filmes

\begin{tabular}{ccc}
\hline Variáveis & Nível inferior $(-)$ & Nível superior $(+)$ \\
\hline A & Concentração de $2 \%(\mathrm{~m} / \mathrm{v})$ & Concentração de $3 \%(\mathrm{~m} / \mathrm{v})$ \\
& de alginato & de alginato \\
B & Tamanho nano menor & Tamanho nano maior \\
& $(15.000 \mathrm{rpm})$ & $(12.000 \mathrm{rpm})$ \\
C & Secagem a $30^{\circ} \mathrm{C}$ & Secagem a $45^{\circ} \mathrm{C}$ \\
\hline
\end{tabular}

No experimento de planejamento fatorial foram produzidos 8 filmes para cada óleo essencial trabalhado (limão, copaíba e melaleuca), chegando ao total de 24 filmes. Depois de produzidos e de avaliados subjetivamente, os filmes foram classificados como: excelente, bom ou deficiente.

Foi possível observar no experimento de avaliação subjetiva, não apenas para o filme de copaíba mostrado na Tabela 3, mas também para os filmes de melaleuca e limão, que os melhores resultados de continuidade, homogeneidade e manuseabilidade foram dos filmes produzidos a partir do experimento 6 .

Observa-se, também, na Tabela 3, que a partir do experimento 5 começa a aparecer resultados com classificação excelente, já os experimentos de 1 a 4 apresentaram resultados mais deficientes.

Sendo assim, através desses resultados foi possível classificar as melhores condições para a produção dos melhores filmes, escolhendo os melhores filmes para serem caracterizados, ou seja, os mais contínuos, homogêneos e de boa manuseabilidade.

Tabela 3. Avaliação subjetiva do filme com óleo de copaíba

\begin{tabular}{ccccccc}
\hline & A & B & C & Continuidade & Homogeneidade & $\begin{array}{c}\text { Manuseabili- } \\
\text { dade }\end{array}$ \\
\hline 1 & + & + & + & $\bullet$ & $\bullet$ & $\bullet$ \\
2 & - & + & + & $\bullet$ & $\bullet$ & $\bullet$ \\
3 & + & - & + & $\bullet$ & $\bullet$ & $\bullet$ \\
4 & - & - & + & $\bullet$ & $\bullet$ & $\bullet$ \\
5 & + & + & - & $\bullet$ & $\bullet$ & $\bullet$ \\
6 & - & + & - & $\bullet$ & $\bullet$ & $\bullet$ \\
7 & + & - & - & $\bullet$ & $\bullet$ & $\bullet$ \\
8 & - & - & - & $\bullet$ & $\bullet$ & $\bullet$ \\
\hline
\end{tabular}

$\checkmark$ excelente; $\bullet$ boa; $\boldsymbol{\square}$ deficiente

Como apresentado na Figura 4, ocorreu um aumento significativo no valor de permeabilidade de vapor de água (WVP) para os filmes de alginato de sódio com óleo essencial de copaíba, limão e melaleuca em relação aos filmes de alginato de sódio puro. Kavoosi et al., ${ }^{9}$ que produziram filmes de gelatina com óleo de Zataria, afirmaram que o valor de WVP depende da interação hidrófilo-hidrófobo presente no filme.

Os filmes 7 tiveram maiores valores de WVP, o que pode estar relacionado com o tamanho das partículas de nanoemulsão e com 


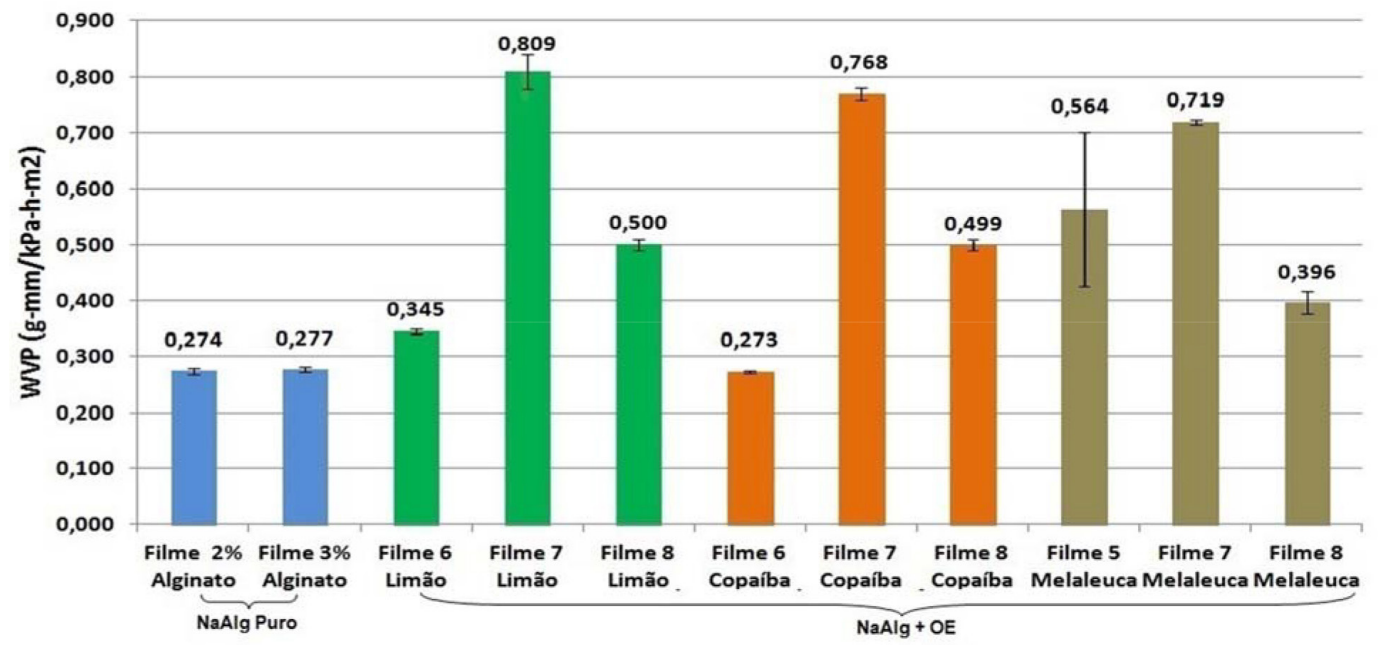

Figura 4. Valores de WVP para os filmes de NaAlg puro e para os filmes de NaAlg com OE de limão, melaleuca e copaíba

a concentração de alginato presente no filme, visto que estes filmes foram produzidos com tamanhos menores de partículas de nanoemulsões e maiores concentrações de alginato $(3 \% \mathrm{~m} / \mathrm{v})$.

As partículas de nanoemulsões possuem uma parte hidrofóbica (lipofílica) que interage com o óleo e uma parte hidrofílica que interage com a água durante sua formação. Depois dos filmes produzidos e secos a parte hidrofílica deixa de interagir com a água que evapora e passa a interagir com as cadeias de alginato através de ligações de hidrogênio. Essa interação com o alginato ocorre mais abundantemente em filmes com nanoemulsões de partículas menores do que com nanoemulsões de partículas maiores devido a sua melhor distribuição ao longo da matriz de alginato.

A Figura 5 mostra essa forte interação das partículas de nanoemulsões menores com a matriz de alginato, fazendo com que estas "ignorem" o vapor de água que passa pela matriz, facilitando sua passagem e aumentando a WVP.

$\mathrm{O}$ aumento da concentração de alginato na produção dos filmes também facilita a passagem de vapor de água e aumenta significativamente os valores de WVP. Isso ocorre porque uma matriz com

a)

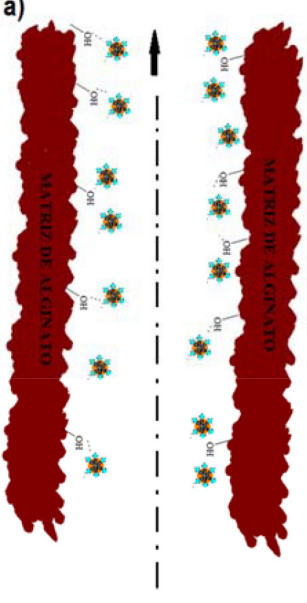

Vapor permeante

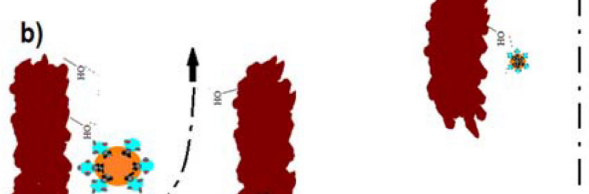

Vapor permeante menos grupamentos polares, ou seja, menor concentração de alginato, pode não interagir com todas as partículas de nanoemulsões presentes nos filmes, fazendo com que elas segurem a passagem de vapor de água. Entretanto, aumentando a concentração de alginato, aumenta-se também a quantidade de grupamentos polares, os quais interagem com as nanoemulsões que antes estavam livres. Essa interação faz com que os vapores de água passem livremente, aumentando os valores de WVP como pode ser visto na Figura 6. a)

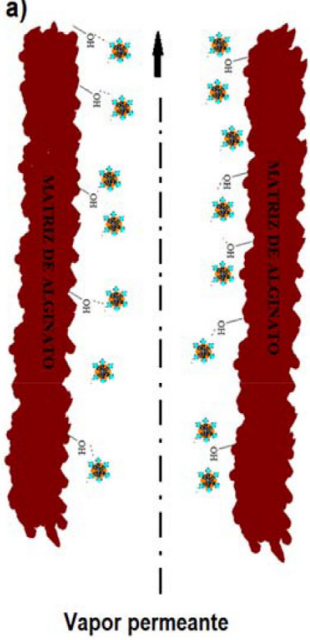

b)

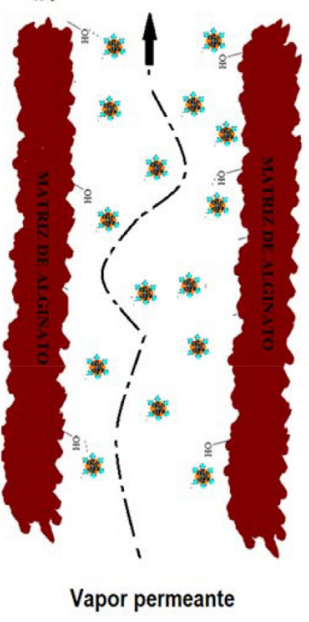

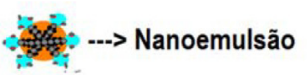

Figura 6. Desenho representativo do efeito da porcentagem de alginato no valor de WVP: (a) $2 \% \mathrm{~m} / \mathrm{v}$ de alginato e (b) $3 \% \mathrm{~m} / \mathrm{v}$ de alginato

Através das imagens reproduzidas pelo MEV é possível observar a presença de nanoemulsões dos óleos incorporados na matriz de alginato. Os filmes 7 de melaleuca (Figura 7 (b)) apresentam rugosidade e aglomerados não observados na Figura 7 (b) do filme de alginato puro. Esses aglomerados e rugosidades são efeitos das nanoemulsões presentes no filme.

\section{CONCLUSÃO}

Através da análise de WVP ficou evidente que os filmes que tiveram melhor permeabilidade ao vapor de água foram os filmes 7
Figura 5. Desenho representativo das interações hidrófilo-hidrófobo das nanoemulsões de OE na matriz de alginato: (a) partículas de nanoemulsões maiores e (b) partículas de nanoemulsões menores 


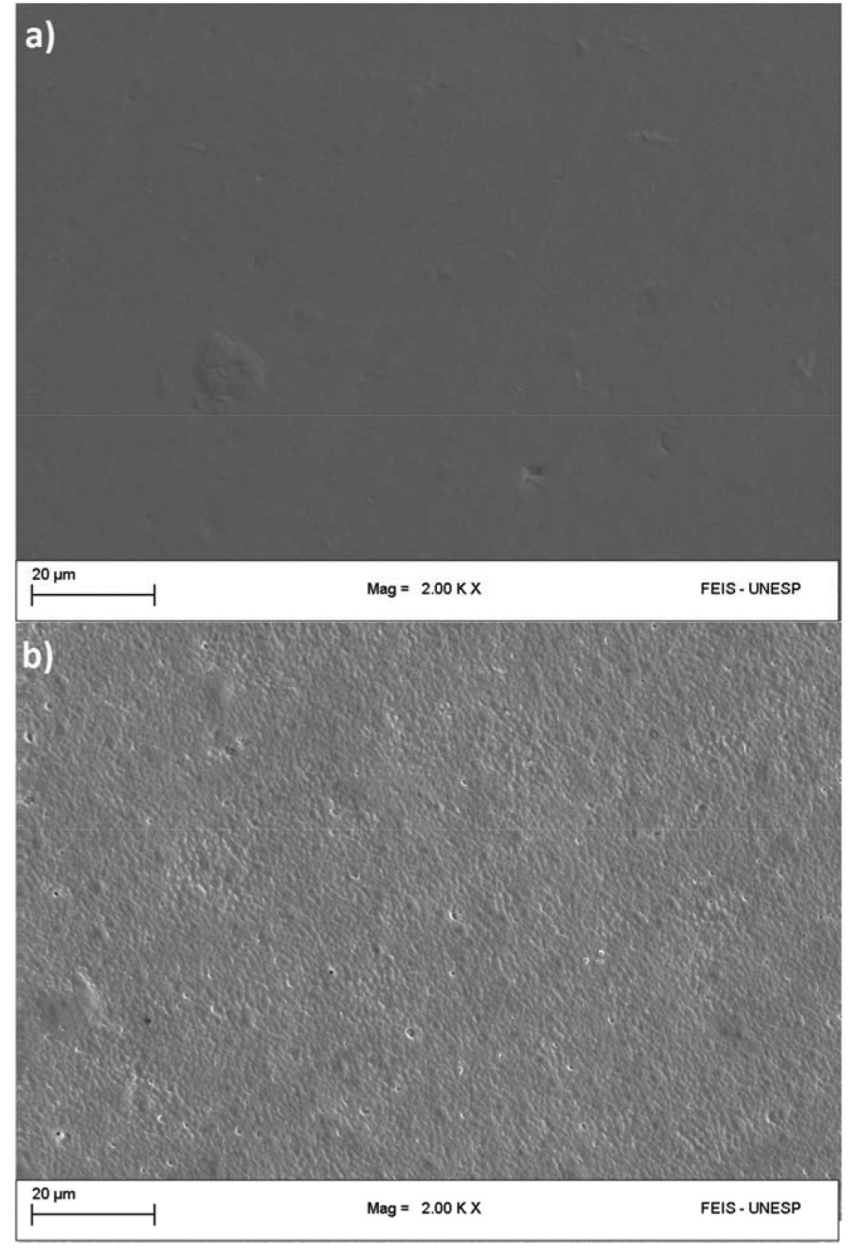

Figura 7. Imagens da Microscopia Eletrônica de Varredura (MEV): (a) Filme controle de alginato de sódio e (b) Filme 7 melaleuca (nanoemulsão de tamanho de partícula menor)

de todos os óleos estudados. Isso está relacionado ao tamanho das partículas de nanoemulsões presente nos filmes, que eram os menores, como observado na análise de tamanho de partícula, e também à maior concentração de alginato. O planejamento fatorial utilizado para a produção dos filmes mostrou-se muito eficiente, uma vez que foi possível obter as melhores condições de preparação dos mesmos. A presença de nanoemulsões é evidenciada na imagem produzida pelo MEV, na o filme com nanoemulsão apresenta rugosidade e aglomerados não observados no filme de alginato puro. Assim, apesar de ser necessária a realização de mais análises, os filmes produzidos têm uma característica que é excelente para uma futura aplicação como curativos, que é a boa WVP. Isso é possível devido à aplicação do óleo na forma de nanoemulsão, a qual tem se mostrado um diferencial neste trabalho.

\section{AGRADECIMENTOS}

Os autores agradecem a CNPq, UNESP, CAPES, FAPESP e EMBRAPA.

\section{REFERÊNCIAS}

1. Sellimi, S.; Younes, I.; Ayed, H. B.; Maalej, H.; Montero, V.; Rinaudo, M.; Dahia, M.; Mechichi, T.; Hajji, M.; Nasri, M.; Int. J. Biol. Macromol. 2015, 72, 1358.

2. Venkatesan, J.; Bhatnagar, I.; Manivasagan, P.; Kang, K.; Kim, S.; Int. J. Biol. Macromol. 2015, 72, 269.

3. Zahran, M. K.; Ahmed, H. B.; El-Rafie, M. H.; Carbohydr. Polym. 2014, $108,145$.

4. Passe, E. R. G.; Blaine, G.; J. -Lancet. 1948, 252, 651.

5. Liao, N.; Unnithan, A. R.; Joshi, M. K.; Tiwari, A. P.; Hong, S. T.; Park, C.; Kim, C. S.; Colloids Surf., A 2015, 469, 194.

6. Mogoşanu, G. D.; Grumezescu, A. M.; Int. J. Pharm. 2014, 463, 127.

7. Liakos, I.; Rizzello, L.; Scurr, D. J.; Pompa, P. P.; Bayer, I. S.; Athanassiou, A.; Int. J. Pharm. 2014, 453, 137.

8. Silva, J. K. R.; Pinto, L. C.; Burbano, R. M. R.; Montenegro, R. C.; Guimarães, E. F.; Andrade, E. H. A.; Maia, J. G. S.; Ind. Crops Prod. 2014, 58, 55.

9. Kavoosi, G.; Rahmatollahi, A.; Dadfar, S. M. M.; Purfard, A. M.; $L W T$ -- Food Sci. Technol. 2014, 57, 556.

10. Lee, C.; Chen, L.; Chen, L.; Chang, T.; Huang, C.; Huang, M.; Wang, C.; J. Food Drug Anal. 2013, 21, 169.

11. Thomsen, N. A.; Hammer, K. A.; Riley, T. V.; Belkum, A. V.; Carson, C. F.; Int. J. Antimicrob. Agents 2013, 41, 343.

12. Forrer, M.; Kulik, E. M.; Filippi, A.; Waltimo, T.; Arch. Oral Biol. 2013, $58,10$.

13. Hammer, K. A.; Int. J. Antimicrob. Agents 2015, 45, 106.

14. Dias, D. O.; Colombo, M.; Kelmann, R. G.; Kaiser, S.; Lucca, L. G.; Teixeira, H. F.; Limberger, R. P.; Veiga Jr., V. F.; Koester, L. S.; Ind. Crops Prod. 2014, 59, 154.

15. Bonan, R. F. B.; Bonan, P. R. F.; Batista, A. U. D.; Sampaio, F. C.; Albuquerque, A. J. R.; Moraes, M. C. B.; Mattoso, L. H. C.; Glenn, G. M.; Medeiros, E. S.; Oliveira, J. E.; Mater. Sci. Eng., Proc. Conf. 2015, 48,372 .

16. Fagg, C. W.; Lughadha, E. N.; Milliken, W.; Hind, D. J. N.; Brandão, M. G. L.; J. Ethnopharmacol. 2015, 161, 18.

17. Tao, N.; Jia, L.; Zhou, H.; Food Chem. 2014, 153, 265.

18. Al-jabri, N. N.; Hossain, M. A.; Beni-Suef University Journal of Basic and Applied Sciences 2014, 3, 247.

19. Ahmad, N.; Ramsch, R.; Llinàs, M.; Solans, C.; Hashim, R.; Tajuddin, H. A.; Colloids Surf., B 2014, 115, 267.

20. American Society for Testing and Materials; Standard test method for water vapor transmission of materials. ASTM E96-80, 1980.

21. Monterrey, E. S.; Sobral, P. J. A.; Ciênc. Tecnol. Aliment. 1999, 19, 294.

22. Moura, M. R.; Aouada, F. A.; Avena-Bustillos, R. J.; McHugh, T. H.; Krochta, J. M.; Mattoso, L. H. C.; J. Food Eng. 2009, 92, 448.

23. Schaffazick, S. R.; Guterres, S. S.; Quim. Nova 2003, 26, 726.

24. Roland, I.; Piel, G.; Delattre, L.; Evrard, B.; Int. J. Pharm. 2003, 263, 85.

25. Liu, W.; Sun, D.; Li, C.; Liu, Q.; Jian Xu. J.; Colloid Interface Sci. 2006, $303,557$. 